International Journal of Wireless \& Mobile Networks ( IJWMN ), Vol.2, No.3, August 2010

\title{
Parameterized AfFect of Transmission- RANGE ON LOST OF NETWORK CONNECTIVITY (LNC) OF WIRELESS SENSOR NETWORKS
}

\author{
Amer A. Al-Rahayfeh, Muder M. Almi'ani, and Abdelshakour A. Abuzneid \\ Computer Science and Engineering Department \\ University Of Bridgeport, Bridgeport, CT 06601, USA \\ \{ aalrahay, malmiani, abuzneid \} @bridgeport.edu
}

\begin{abstract}
Wireless Sensor Networks, referred to as WSNs, are made up of various types of sensor nodes. Recent developments in micro electro-mechanical technology have given rise to new integrated circuitry, microprocessor hardware and nano technology, wireless technology, and advanced networking routing protocols. Hospitals and health service facilities, the armed forces, and even residential customers represent a potential huge market for these devices. The problem is that existing sensor network nodes are incapable of providing the support needed to maximize usage of wireless technology.

For this reason, there are many novel routing protocols for the wireless sensor networks proposed recently. One is Hierarchical or cluster-based routing. In this paper, we analyze three different types of hierarchical routing protocols: Low Energy Adaptive Clustering Hierarchy (LEACH), Power-Efficient Gathering in Sensor Information Systems (PEGASIS), and Virtual Grid Architecture (VGA). We tried to analyze the performance of these protocols, including the power consumption and overall network performance. We also compared the routing protocol together. This comparison reveals the important features that need to be taken into consideration while designing and evaluating new routing protocols for sensor networks. The simulation results, using same limited sensing range value, show that PEGASIS outperforms all other protocols while LEACH has better performance than VGA. Furthermore, the paper investigates the power consumption for all protocols. On the average, VGA has the worst power consumption when the sensing range is limited, while VGA is the best when the sensing range is increased. Using homogeneous nodes can greatly prolong sensor network's life time. Also, The network lifetime increases as the number of clusters decreases
\end{abstract}

\section{KEYWORDS}

Energy consumption, network performance, Sensor networks, Routing protocols, LEACH, PEGASIS, VGA, Cluster head.

\section{INTRODUCTION}

Wireless sensor networks (WSNs) are ad hoc networks comprised mainly of small sensor nodes with limited resources (low power, low bandwidth, and low computational and storage capabilities) and one or more base stations (BSs), which are much more powerful nodes that connect the sensor nodes to the rest of the world. WSNs are rapidly emerging as a technology for large-scale, low-cost, automated sensing and monitoring of different environments of interest. Potential WSN applications range from battlefield reconnaissance to environmental protection [1, 2].

These nodes form a network by communicating with each other either directly or through other nodes. One or more nodes will serve as sink(s) that are capable of communicating with the user either directly or through the existing wired networks. The primary component of the network is the sensor, essential for monitoring real world physical conditions such as sound, temperature, humidity, intensity, vibration, pressure, motion, pollutants etc. at different locations. The tiny 
sensor nodes, which consist of sensing, on board processor for data processing, and communicating components, leverage the idea of sensor networks based on collaborative effort of a large number of nodes [3][4] [14].

Generally speaking, the methods of arranging WSN systems can be classified into one of several categories: flat-based routing, hierarchical-based routing, and location based routing. Under the first method, nodes are given equal functionality and utility. The second method, hierarchical based routing, assigns diverse functions in the network. In the location-based system, sensor nodes are utilized to transmit relevant data and information. Assuming that various parameters can be properly organized so as to accommodate current network conditions and available power levels, the system will be "adaptive."

The remainder of the paper is organized as follows: Section 2, we clarify the three hierarchical routing protocols. In Section 3 provides a simulation and the results. Finally, Section 4 concludes the paper.

\section{RELATED WORK}

In general, routing in WSNs can be divided into flat-based routing, hierarchical-based routing, and location-based routing. In this section, we only review three hierarchical routing protocols: LEACH, PEGASIS, and VGA.

Hierarchical or cluster-based routing, originally proposed in wireline networks, are well-known techniques with special advantages related to scalability and efficient communication. As such, the concept of hierarchical routing is also utilized to perform energy-efficient routing in WSNs. In a hierarchical architecture, higher energy nodes can be used to process and send the information while low energy nodes can be used to perform the sensing in the proximity of the target. This means that creation of clusters and assigning special tasks to cluster heads can greatly contribute to overall system scalability, lifetime, and energy efficiency. Hierarchical routing is an efficient way to lower energy consumption within a cluster and by performing data aggregation and fusion in order to decrease the number of transmitted messages to the Base station (BS). Hierarchical routing is mainly two-layer routing where one layer is used to select clusterheads and the other layer is used for routing. However, most techniques in this category are not about routing, rather on "How and when to send or process/aggregate" the information, channel allocation etc., which can be orthogonal to the multihop routing function [5].

\subsection{LEACH protocol}

Heinzelman, et. al. [6] introduced a hierarchical clustering algorithm for sensor networks, called Low Energy Adaptive Clustering Hierarchy (LEACH). LEACH is a cluster-based protocol, which includes distributed cluster formation. He assumes two types of network nodes: a more powerful BS and a larger number of resource-scarce sensor nodes. In WSNs, resource-scarce nodes do not typically communicate directly with the BS for two reasons. One, these nodes typically have transmitters with limited transmission range, and are unable to reach the BS directly. Two, even if the BS is within a node's communication range, direct communication typically demands much higher energy consumption. A more energy efficient alternative takes advantage of one's neighboring nodes as routers. Nodes that are farther away send their messages to intermediate nodes, which then forward them towards the BS in a multi-hop fashion. The problem with this approach is that, even though peripheral nodes actually save energy, the intermediate nodes, which play the role of routers, spend additional energy receiving and transmitting messages, and end up having a shortened lifetime, LEACH assumes every node can directly reach a BS by transmitting with sufficiently high power. However, to save 
energy and avoid the aforementioned problem, LEACH uses a novel type of routing that randomly rotates routing nodes among all nodes in the network. Briefly, LEACH works in rounds and in each round, it uses a distributed algorithm to elect ClusterHeads (CHs) and dynamically cluster the remaining nodes around the CHs. To avoid energy drainage of $\mathrm{CHs}$, they do not remain $\mathrm{CHs}$ forever; nodes take turns in being $\mathrm{CHs}$, and energy consumption spent on routing is thus distributed among all nodes.

In [6], Heinzelman, et. al. said Rounds in LEACH have predetermined duration, and have a setup phase and a steady-state phase. Through synchronized clocks, nodes know when each round starts and ends. The setup consists of three steps. In the advertisement step (Step \# 1), nodes decide probabilistically whether or not to become a $\mathrm{CH}$ for the current round (based on its remaining energy and a globally known desired percentage of $\mathrm{CHs}$ ). Those that will broadcast a message (adv) advertising this fact, at a level that can be heard by everyone in the network. To avoid collision, the CSMA-MAC protocol is used. In the cluster joining step (Step \# 2), the remaining nodes pick a cluster to join based on the largest received signal strength of an adv message, and communicate their intention to join by sending a join req (join request) message using CSMA-MAC. Once the CHs receive all the join requests, the confirmation step (Step \# 3) starts with the $\mathrm{CHs}$ broadcasting a confirmation message that includes a time slot schedule to be used by their cluster members for communication during the steady-state phase. Given that the CHs' transmitters and receivers are calibrated, balanced and geographically distributed, clusters are created.

Once the clusters are set up, the network moves on to the steady-state phase, where actual communication between sensor nodes and the BSs takes place. Each node knows when it is its turn to transmit (Step \# 4), according to the time slot schedule. The CHs collect messages from all their cluster members, aggregate these data, and send the result to the BS (Step \#5). The steady-state phase consists of multiple reporting cycles, and lasts much longer compared to the setup phase.

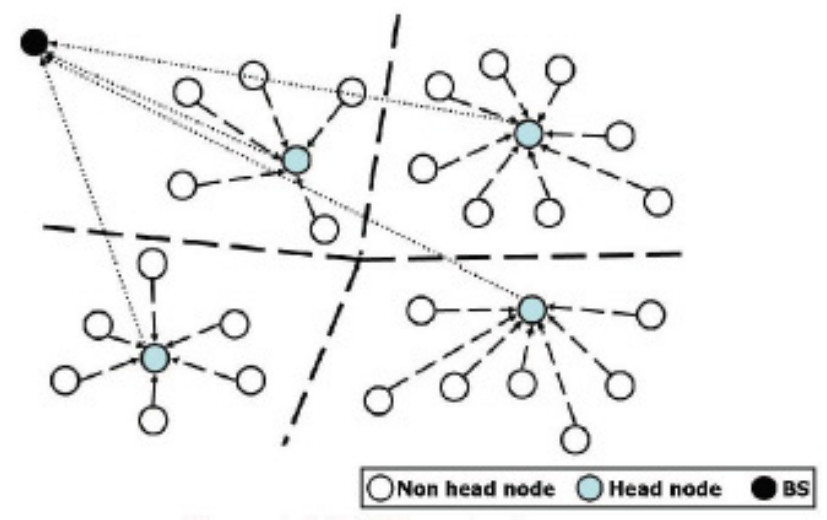

Figure 1. LEACH protocol [13]

\subsection{PEGASIS protocol}

In [7], an enhancement over LEACH protocol was proposed. The protocol, called Powerefficient Gathering in Sensor Information Systems (PEGASIS), is a near optimal chain-based protocol. 
The main idea in PEGASIS is for each node to receive from and transmit to close neighbors and take turns being the leader for transmission to the BS. This approach will distribute the energy load evenly among the sensor nodes in the network. We initially place the nodes randomly in the play field, and therefore, the $\mathrm{i}$-th node is at a random location. The nodes will be organized to form a chain, which can either be accomplished by the sensor nodes themselves using a greedy algorithm starting from some node. Alternatively, the BS can compute this chain and broadcast it to all the sensor nodes.

The PEGASIS protocol runs as follows:

* Chain construction

The PEGASIS protocol performs two steps to construct the chain. In first step, sensor nodes and the base station are self-organized using the greedy algorithm. In second step, the base station broadcast information of the chain to sensor nodes after it performs the process of the chain construction. In the former step, the chain construction is started at the farthest node from the base station.

This step is continued until all nodes are on the chain. At this time, each node uses signal strength to measure the distance with neighbor nodes and then adjusts the signal strength so that only one node can hear its message [8] [9].

* Gathering data

After the PEGASIS protocol performs the process of the chain construction, each node delivers own sensing data to its neighbor node. After receiving these data, the neighbor node aggregates them with their own data and transmits these data to its neighbor node. Each node performs this task that sensing data, it delivered to base station (BS) in turns [8] [9].

The data transmission is performed as shown in Figure 2. The PEGASIS protocol uses a token which contains a small message. The node $\mathrm{c} 2$ is the head node and it will pass a token along the chain to node $\mathrm{c} 0$. Node $\mathrm{c} 0$ will deliver sensing data to node $\mathrm{cl}$. Then node $\mathrm{cl}$ receives these data, fuses with its own data, and transmits these data to node $\mathrm{c} 2$. After node $\mathrm{c} 3$ receives the data, it will pass a token to node $\mathrm{c} 4$, and node $\mathrm{c} 4$ will pass its data toward node $\mathrm{c} 2$ in the same way [8] [9]. In sum, the PEGASIS protocol constructs the chain and each node collects and delivers to its nearest neighbor node. As each node is selected to be head node in turns, the PEGASIS protocol can save the energy remarkably.

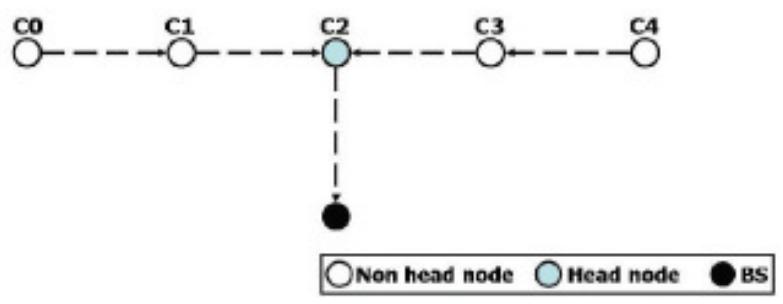

Figure 2. PEGASIS protocol [13] 


\subsection{VGA Protocol}

Virtual Grid Architecture routing (VGA) paradigm is proposed in [10]. The main objective of VGA is to create a fixed rectilinear virtual topology on which the routing and network management functions can be performed easily and efficiently.

1) VGA clusters formation: In VGA clustering, the network area is divided into fixed, disjoint, and regular shape zones. To create a simple rectilinear virtual topology, we select the zones to be square in shape. Each mobile node is a member of one of those zones and its zone membership is determined based on its location in the network area. In homogeneous networks, where all nodes have the same transmission range, $r$, the zone side length $x$ is chosen such that two mobile nodes in adjacent horizontal/vertical zones, and located anywhere in their zones, can communicate with each other directly. Therefore, $x=r / \sqrt{5}$. In heterogeneous networks, nodes have different transmission ranges. For the sake of simplicity, but without loss of generality, assume two different transmission ranges, e.g., $r_{s}$ for Short Range (SR) nodes and $r_{1}$ for Long Range (LR) nodes. Initially, the network area is divided into large zones where the zone side length $\mathrm{x}_{1}$ is chosen as $x_{1}=r_{1} / \sqrt{ } 5$. If the zone has only short range nodes, the zone is further divided into four subzones where each subzone side length $\left(x_{s}\right)$ is computed as $x_{s}=x_{1} / 2$. The number of layers in the virtual topology is determined by the number of transmission ranges in the network [10].

2) Clusterheads Selection: After zoning is finished, a periodic CHs election algorithm is executed in each zone of the VGA. The CH periodicity helps to balance the nodes' load distribution, achieves fairness, and provide fault tolerance against single node failure. A clusterhead may also trigger the clusterhead election algorithm when its energy level falls below a certain threshold or when it leaves its zone early. In each period, a node is selected as a clusterhead based on an eligibility factor $(\mathrm{EF})$.

3) Routing in VGA: In VGA, a clusterhead $(\mathrm{CH})$ communicates only with its vertical and horizontal neighbors directly, and therefore routing is done on virtual rectilinear grid. The extension to diagonal routing, henceforth called Diagonal VGA (D-VGA), is possible but it may complicate routing since the number of potential neighbor zones doubles. This is because the zone side length is reduced to a maximum of $(\mathrm{r} / 2 \sqrt{2})$ per side as opposed to $(\mathrm{r} / \sqrt{ } 5)$ when rectilinear routing is used. Routing over VGA is simple where packets are routed through the set of $\mathrm{CHs}$ and in the associated vertical and horizontal directions only. Hence, a simple packet forwarding scheme can be easily implemented over VGA. One simple packet forwarding scheme can be implemented as follows. The standard four directions (North (N), South(S), West (W), and East (E)) are used for simple packet forwarding in the resulting virtual grid. Those directions can be encoded using a 2-bit representation in the packet header such that (00-01-10-11) correspond to (N-S-E-W) directions, respectively [10].

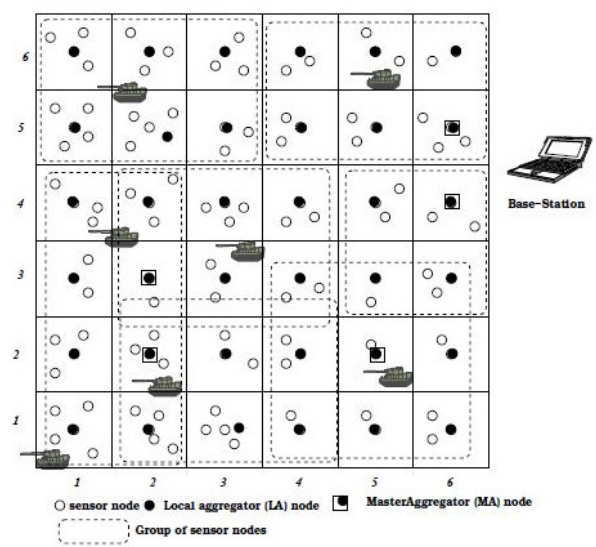

Figure 3. The VGA clustering approach [13] 


\section{SIMULATION AND RESULTS}

In this section, we use Sensoria [11] as our simulation platform. Sensoria is a new and powerful simulator for WSN research. Simulation of LEACH, PEGASIS, and VGA were conducted then followed by a comparison among the different techniques.

Three scenarios are tested to measure the performance, as follows:

3.1 Nodes are Homogeneous:

3.1.1 The nodes are Homogeneous with short transmission range

3.1.2 The nodes are Homogeneous with long transmission range

3.2 Nodes are Heterogeneous

3.2.1 The nodes are Heterogeneous with short transmission range

3. 2.2 The nodes are Heterogeneous with long transmission range

3.3 Percentage of cluster head

These three scenarios are tested for the Loss of network connectivity (LNC). We will report the network- life- time at a round when a sensor node becomes isolated (all its neighbors ran out of energy), i.e. the network is not fully connected. We prep our testing by simulating LNC with ascending transmission-range for three famous protocols.

Table 1. The network lifetime vs transmission-range

\begin{tabular}{|l|l|l|l|}
\hline Range $(\mathrm{m})$ & LEACH & PEGASIS & VGA \\
\hline 20 & 1557 & 3165 & 70 \\
\hline 40 & 1591 & 2068 & 5615 \\
\hline 60 & 1626 & 2171 & 5690 \\
\hline 80 & 1646 & 2190 & 5750 \\
\hline 100 & 2278 & 2278 & 5808 \\
\hline
\end{tabular}

Figure 4 shows the results:

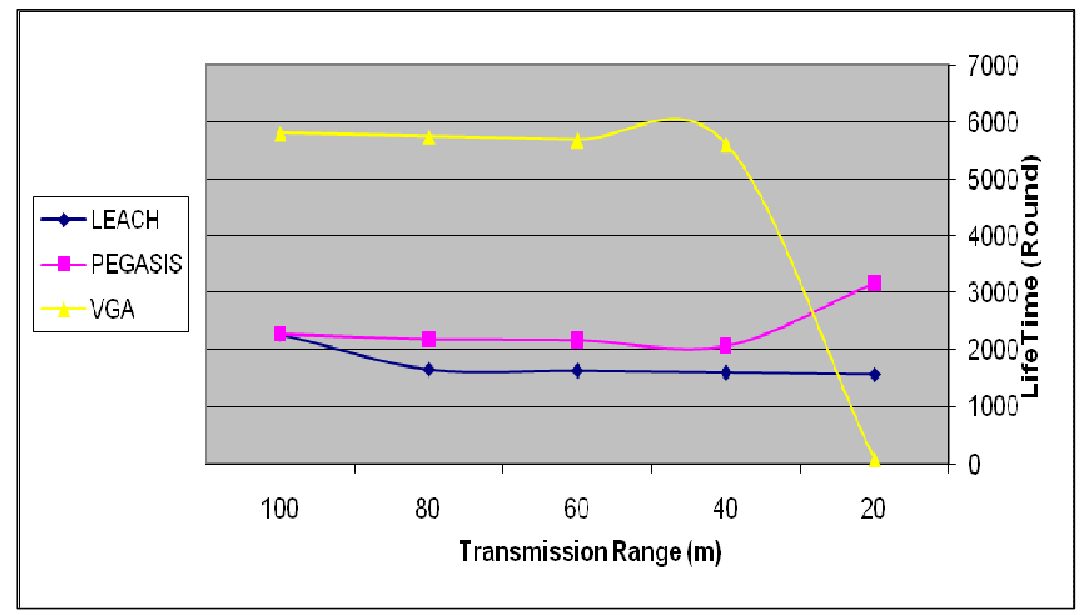

Figure 4. Range vs. Life time 


\subsubsection{The nodes are homogeneous with short transmission range}

To evaluate the performance of the three hierarchal routing protocols, we simulated PEGASIS, LEACH and VGA using a 100 fixed homogeneous (i.e. the same initial energy) sensor nodes. The nodes are initially equipped with $0.5 \mathrm{j} /$ node and scattered randomly in the grid. The BS is located at $(25,150)$ in a $50 \mathrm{~m} \times 50 \mathrm{~m}$ field. Transmission and sensing range are $15 \mathrm{~m}$ and $20 \mathrm{~m}$ respectively.

We assume that each sensor node generates one data packet per time unit to be transmitted to the BS. For simplicity, we refer to each time unit as a round.

Physical parameters are the same of all three protocols: Transmission speed $100 \mathrm{bit} / \mathrm{s}$, Network bandwidth $5000 \mathrm{bit} / \mathrm{s}$ and Data packet processing delay $0.1 \mathrm{~ms}$. Protocol parameters for LEACH are: Cluster type Dynamic, CHs percentage 5\% and $\mathrm{CHs}$ selection cycle 1sec. No special parameters for PEGASIS. VGA parameters are: Local Aggregation (Las) and Master Aggregation MAs selection cycle 1sec.

\section{- Results and Discussion}

In the first scenario we ran the simulation to determine the number of rounds of communication until the network lose its connectivity using LEACH, PEGASIS, and VGA when the transmission range is $20 \mathrm{~m}$. Our simulation shows the following results:

1. Lifetime of network: 3165,1557 , and 70 rounds for PEGASIS, LEACH, and VGA respectively, figure 5 shows the network life time.

2. Figure 6 shows the total network energy versus time.

3. The number of failed nodes are 52 at the last period in PEGASIS, 50 in LEACH and 9 in VGA; figure 7 shows the number of failed nodes versus time for the three routing algorithms.

Therefore, you can conclude that PEGASIS achieves:

- Approximately $2 \mathrm{x}$ the number of rounds compared to LEACH.

- Approximately $45 x$ the number of rounds compared to VGA.

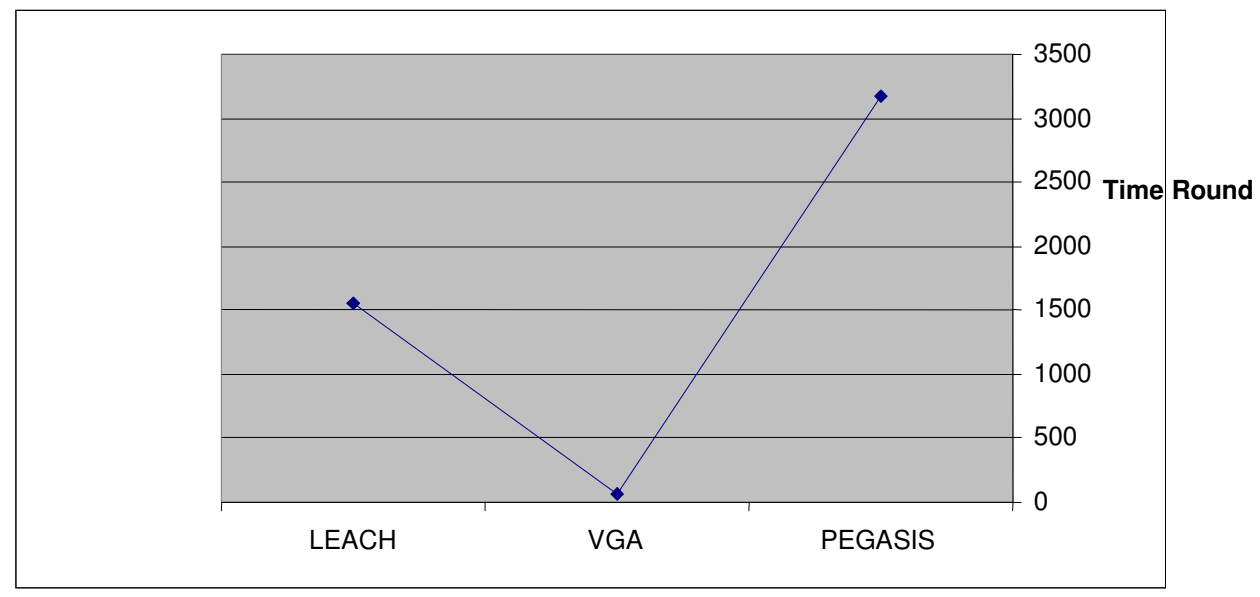

Figure 5. Network life time 

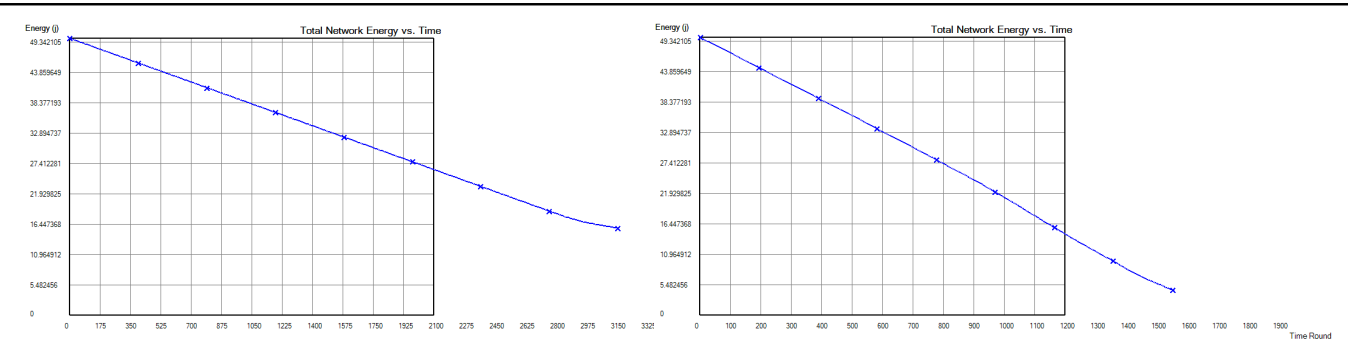

A. PEGASIS total network energy

B. LEACH total network energy

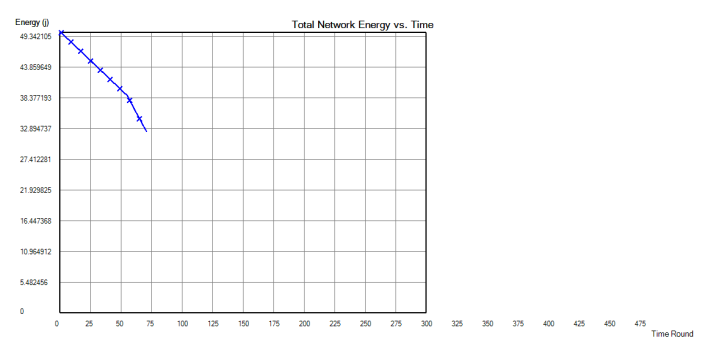

C. VGA total network energy

Figure 6. Total network energy

\subsubsection{Nodes are homogeneous with long transmission range}

Again, we ran the simulations to determine the number of rounds of communication when the network loss its connectivity, where this time the transmission range is $100 \mathrm{~m}$. Our simulations show the following results:

1. Lifetime of the network: 5808, 2278, and 1700 rounds for VGA, PEGASIS and LEACH respectively, figure 8 shows the network life time.

2. Figure 9 shows the total network energy versus time.

3. The number of failed nodes became 99 at the last period in PEGASIS, 93 in LEACH and 69 in VGA; figure 10 shows the number of failed nodes versus time. 
International Journal of Wireless \& Mobile Networks ( IJWMN ), Vol.2, No.3, August 2010

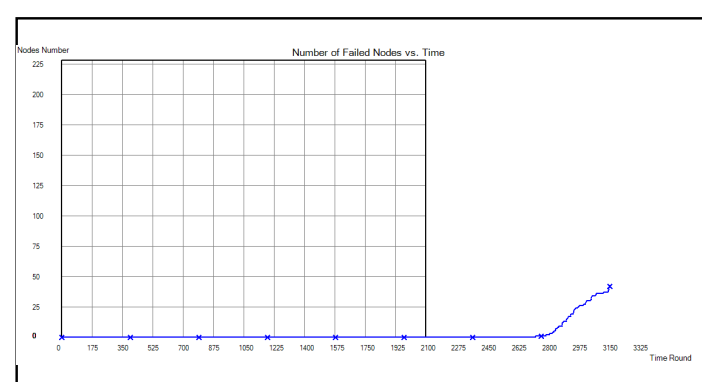

A. PEGASIS failed nodes

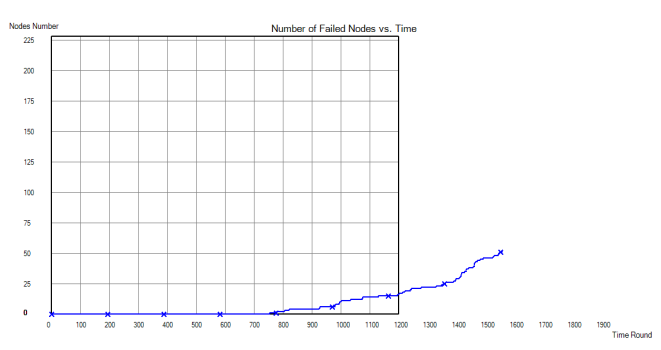

B. LEACH failed nodes

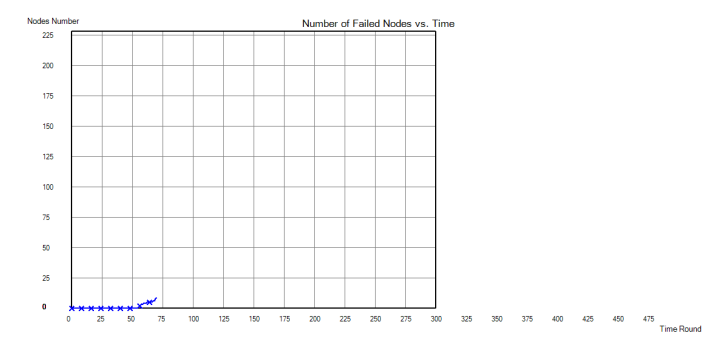

C. VGA failed nodes

Figure 7. The number of failed nodes

Therefore, you can conclude that VGA achieves:

- Approximately $2 \mathrm{x}$ the number of rounds compared to PEGASIS.

- Approximately $3.5 x$ the number of rounds compared to LEACH.

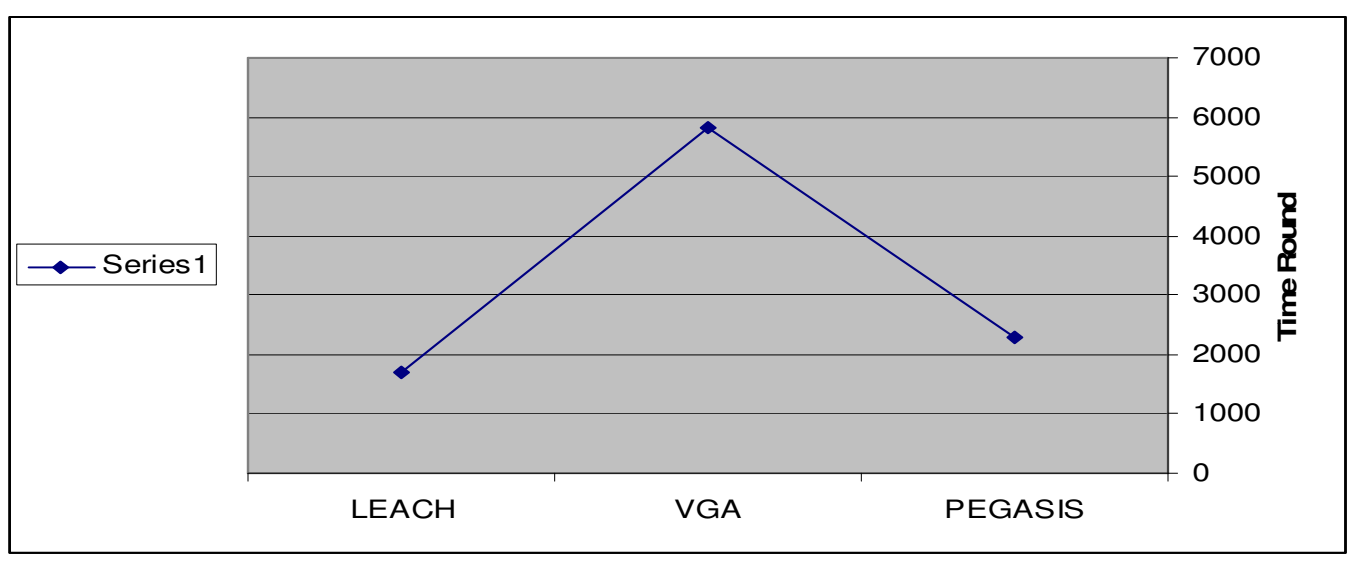

Figure 8. Network life time 
International Journal of Wireless \& Mobile Networks ( IJWMN ), Vol.2, No.3, August 2010
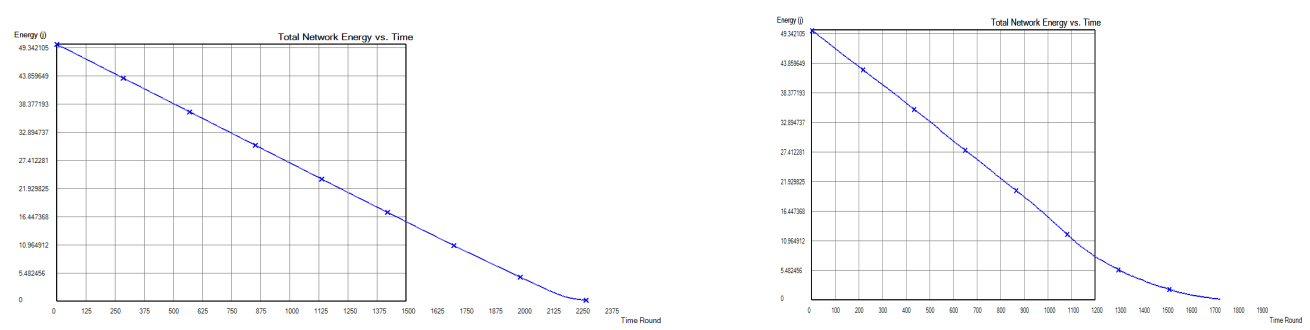

A. PEGASIS total network energy

B. LEACH total network energy

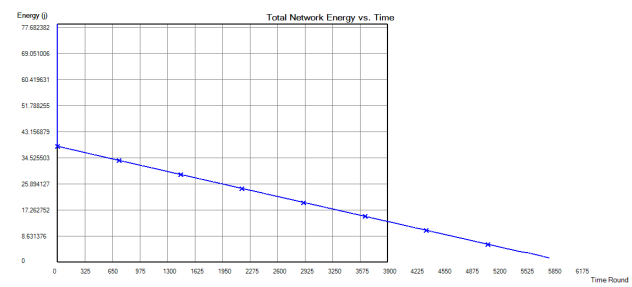

C. VGA total network energy

Figure 9. Total network energy
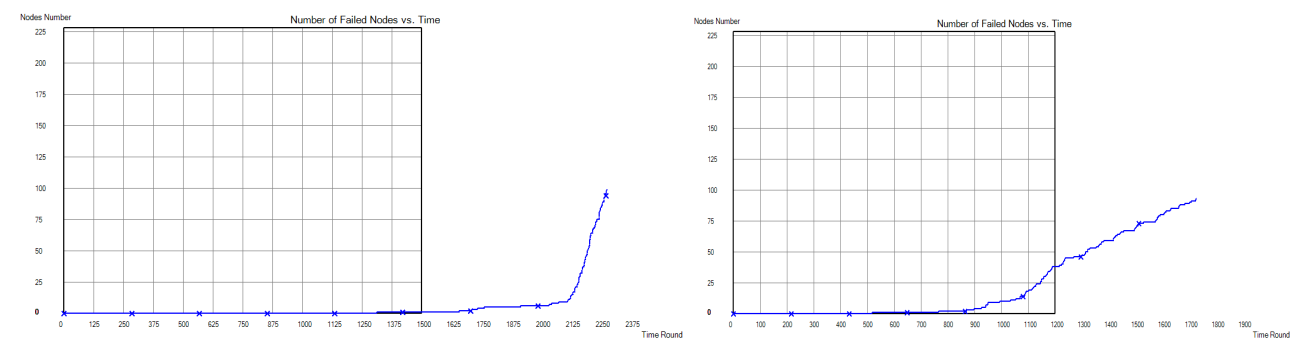

A. PEGASIS failed nodes

B. LEACH failed nodes

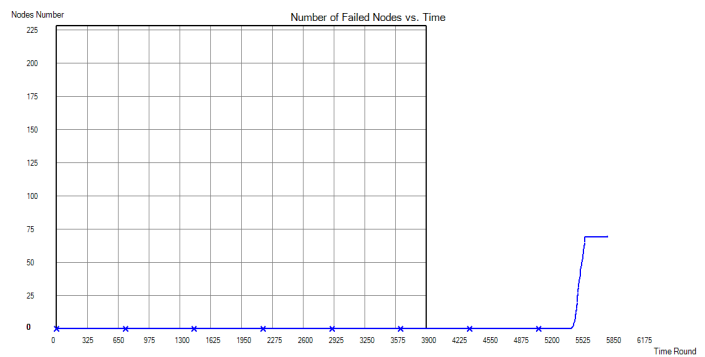

C. VGA failed nodes

Figure 10. The number of failed nodes 


\subsubsection{Nodes are heterogeneous with short transmission range}

We ran the simulation to determine the number of rounds of communication before the network lose its connectivity. However, the nodes are heterogeneous and the transmission range is $20 \mathrm{~m}$. Our simulation shows the following results:

1. Lifetime of the network: 2482,1414 , and 42 rounds for PEGASIS, LEACH and VGA respectively. Figure 11 shows the network life time.

2. Figure 12 shows the total network energy versus time.

3. The number of failed nodes are 69 at the last round in PEGASIS, 76 in LEACH and 4 in VGA; figure 13 shows the number of failed nodes versus time for the three routing algorithms.

We conclude that PEGASIS achieves:

- Approximately $1.8 \mathrm{x}$ the number of rounds compared to LEACH.

- Approximately 59x the number of rounds compared to VGA.

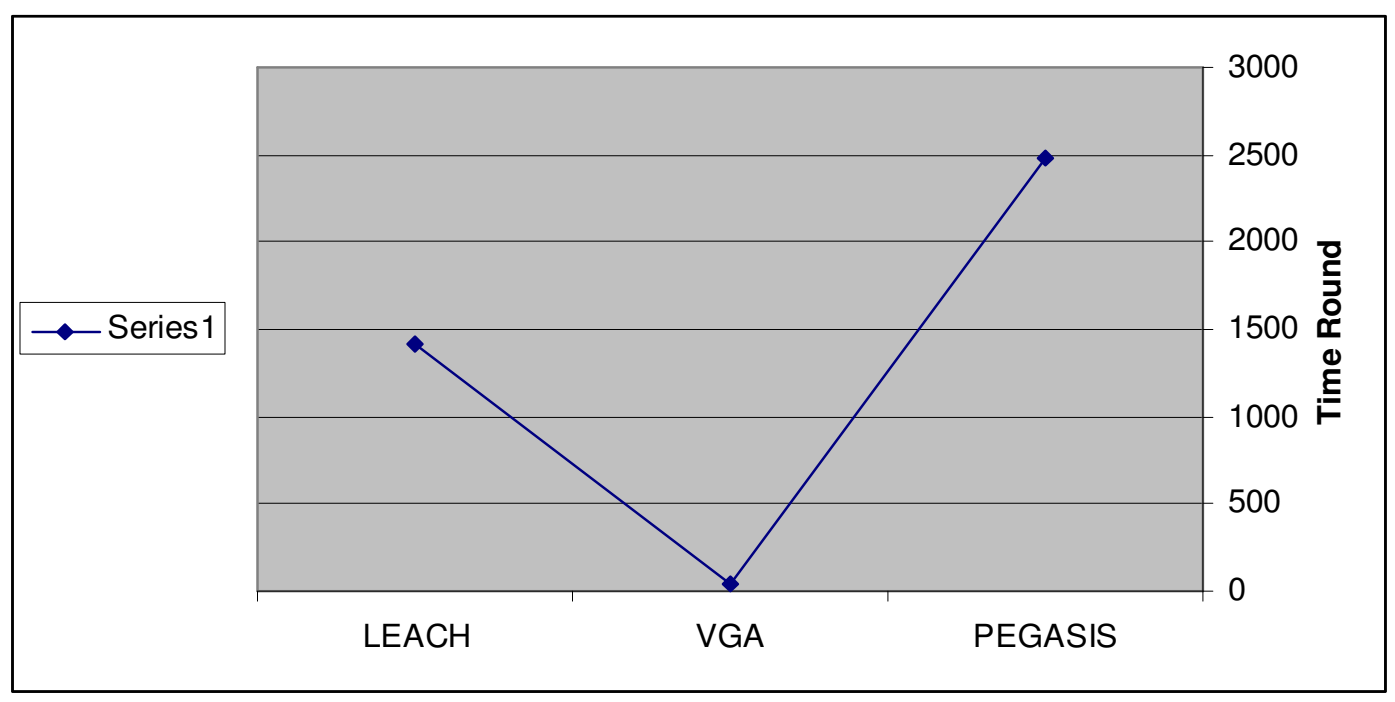

Figure 11. Network life time 
International Journal of Wireless \& Mobile Networks ( IJWMN ), Vol.2, No.3, August 2010
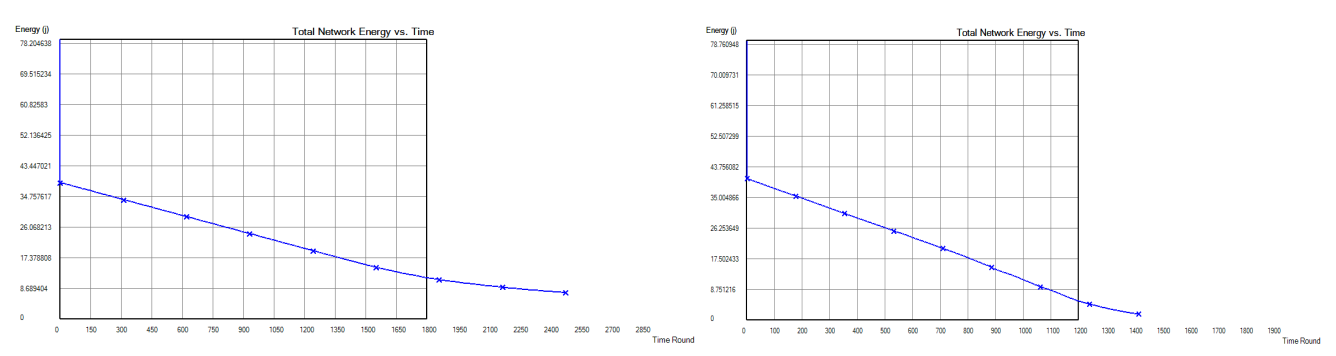

A. PEGASIS total network energy $\quad$ B. LEACH total network energy

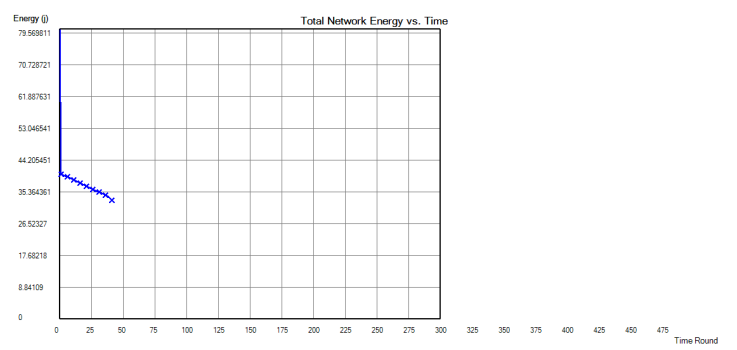

C. VGA total network energy

Figure 12. Total network energy

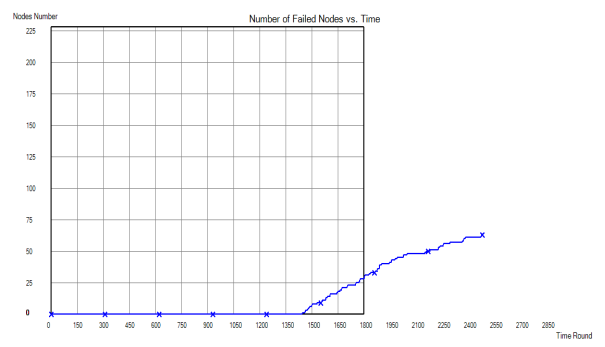

A. PEGASIS failed nodes

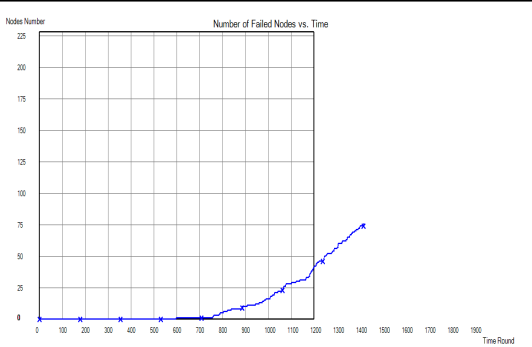

B. LEACH failed nodes

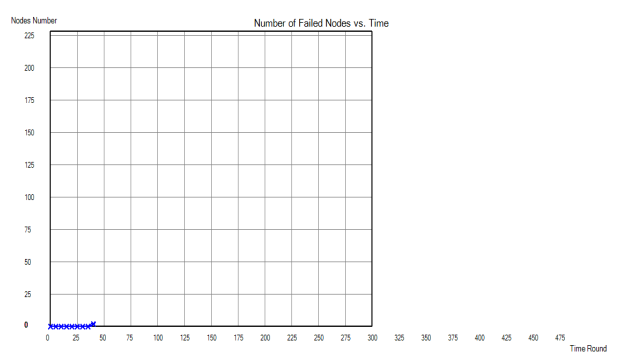

C. VGA failed nodes

Figure 13. The number of failed nodes 


\subsubsection{Nodes are heterogeneous with long transmission range}

We ran the simulation to determine the number of rounds of communication before the network lose its connectivity, where this time the nodes are heterogeneous and the transmission range is $100 \mathrm{~m}$. Our simulations show the following results:

1. Lifetime of network are 4448, 1925, and 1470 rounds for VGA, PEGASIS and LEACH respectively, figure 14 shows the network life time.

2. Figure 15 shows the total network energy versus time.

3. The number of failed nodes became 100 at the last round in PEGASIS, 95 in LEACH and 2 in VGA; figure 16 shows the number of failed nodes versus time.

Therefore, you can conclude that VGA achieves:

- Approximately $2.3 x$ the number of rounds compared to PEGASIS.

- Approximately $3 x$ the number of rounds compared to LEACH.

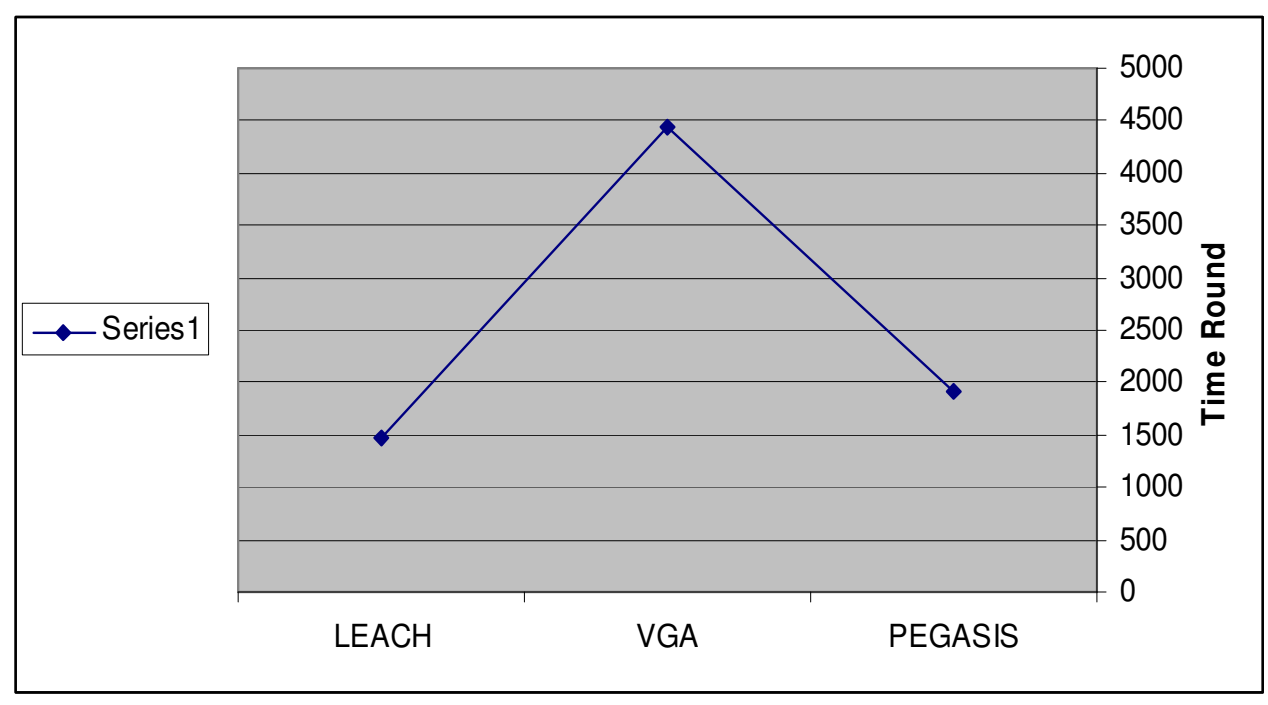

Figure 14. Network life time

Table 2 and Table 3 summarizes the results. We conclude that using PEGASIS may greatly prolong sensor network's-life-time when the transmission range is limited. VGA saves more energy than other protocols when the transmission range is farther. Using homogeneous nodes achieves a better network-life-time when compared to heterogeneous network. 
International Journal of Wireless \& Mobile Networks ( IJWMN ), Vol.2, No.3, August 2010

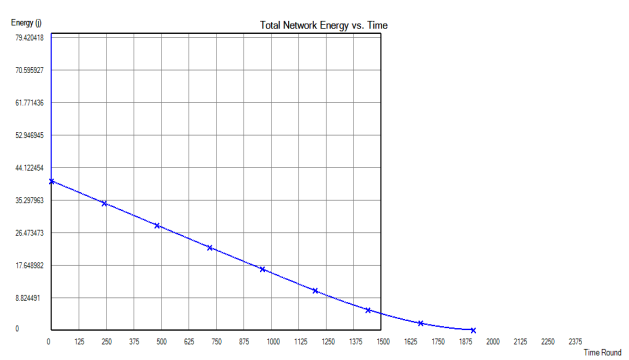

A. PEGASIS total network energy

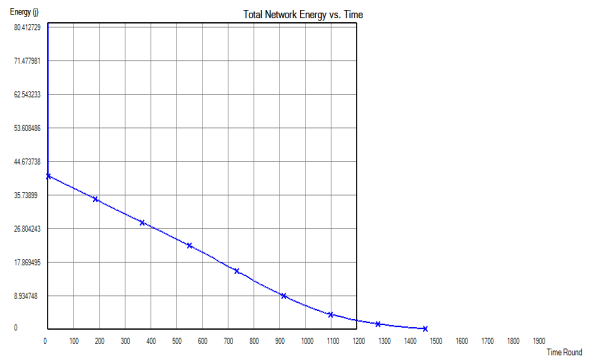

B. LEACH total network energy

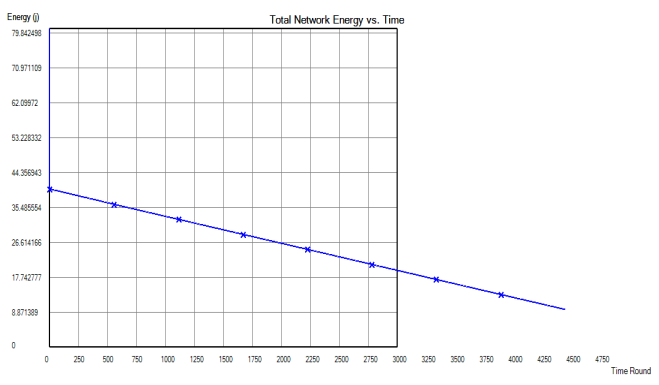

C. VGA total network energy

Figure 15. Total network energy
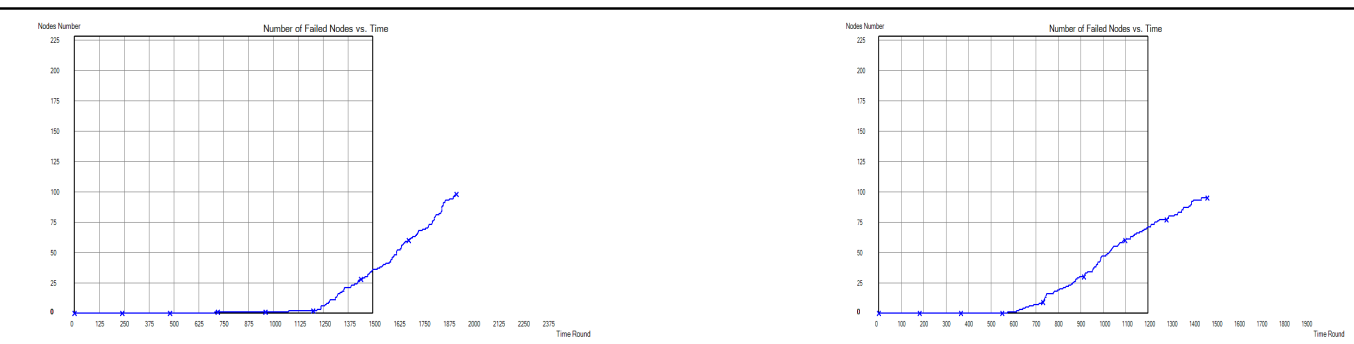

A.PEGASIS failed nodes

\section{B.LEACH failed nodes}

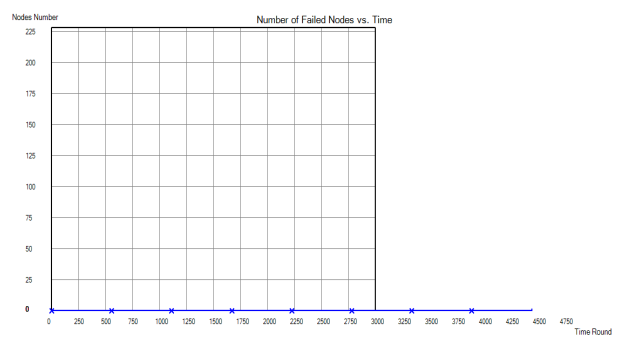

C. VGA failed nodes

Figure 16. The number of failed nodes 


\subsection{Percentage of cluster head}

In this scenario we ran the simulation to determine the number of rounds of communication before the network lose its connectivity. We used different number of clusterheads with each node having the same initial energy level and transmission range which are 0.5 Joule and $15 \mathrm{~m}$ respectively. Our simulations show the following results in Table 3.

As can be seen from the Table 4 and Figure 17, the network lifetime increases as the number of clusters decreases as long as we guarantee connection to BS. This might be due to the observation that as the number of clusters decreases, the route length to BS (in terms of number of hops) will also decrease. Hence, the number of transmissions and receptions of data will also be reduced.

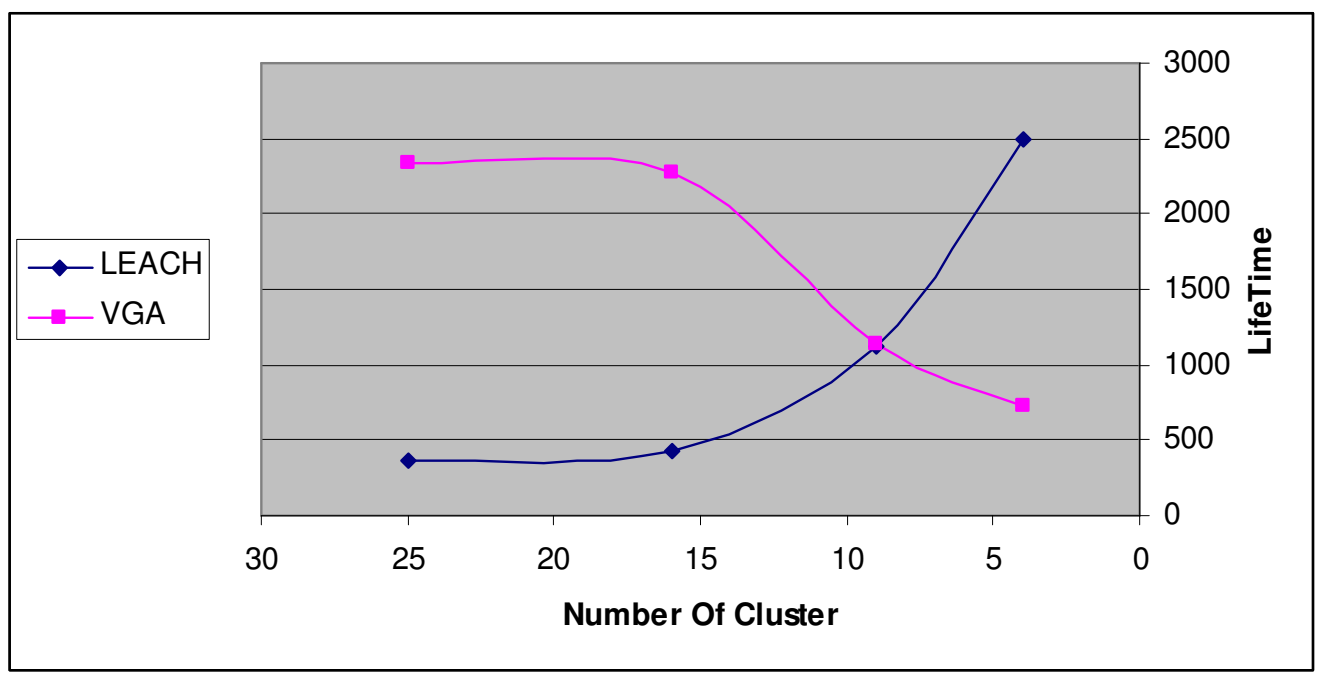

Figure 17. Network life time VS Percentage of Cluster Heads

Table 2. The network lifetime and number of failed node when the nodes are homogeneous

\begin{tabular}{|c|c|c|c|c|c|c|c|c|c|c|c|}
\hline \multicolumn{12}{|c|}{ Homogeneous } \\
\hline \multicolumn{6}{|c|}{ Short transmission range $(15 \mathrm{~m})$} & \multicolumn{6}{|c|}{ long transmission range $(100 \mathrm{~m})$} \\
\hline \multicolumn{2}{|c|}{ PEGASIS } & \multicolumn{2}{|c|}{ LEACH } & \multicolumn{2}{|c|}{ VGA } & \multicolumn{2}{|c|}{ PEGASIS } & \multicolumn{2}{|c|}{ LEACH } & \multicolumn{2}{|c|}{ VGA } \\
\hline $\begin{array}{l}\text { Lifeti } \\
\text { me } \\
\text { (Roun } \\
\text { d) }\end{array}$ & $\begin{array}{l}\text { \# OF } \\
\text { FAIL } \\
\text { ED } \\
\text { NOD } \\
\text { ES }\end{array}$ & $\begin{array}{l}\text { Lifeti } \\
\text { me } \\
\text { (Roun } \\
\text { d) }\end{array}$ & $\begin{array}{l}\text { \# OF } \\
\text { FAIL } \\
\text { ED } \\
\text { NOD } \\
\text { ES }\end{array}$ & $\begin{array}{l}\text { Lifeti } \\
\text { me } \\
\text { (Roun } \\
\text { d) }\end{array}$ & $\begin{array}{l}\# \text { OF } \\
\text { FAIL } \\
\text { ED } \\
\text { NOD } \\
\text { ES }\end{array}$ & $\begin{array}{l}\text { Lifeti } \\
\text { me } \\
\text { (Roun } \\
\text { d) }\end{array}$ & $\begin{array}{l}\text { \# OF } \\
\text { FAIL } \\
\text { ED } \\
\text { NOD } \\
\text { ES }\end{array}$ & $\begin{array}{l}\text { Lifeti } \\
\text { me } \\
\text { (Roun } \\
\text { d) }\end{array}$ & $\begin{array}{l}\# \text { OF } \\
\text { FAIL } \\
\text { ED } \\
\text { NOD } \\
\text { ES }\end{array}$ & $\begin{array}{l}\text { Lifeti } \\
\text { me } \\
\text { (Roun } \\
\text { d) }\end{array}$ & $\begin{array}{l}\# \text { OF } \\
\text { FAIL } \\
\text { ED } \\
\text { NOD } \\
\text { ES }\end{array}$ \\
\hline 3165 & 52 & 1557 & 50 & 70 & 9 & 2278 & 99 & 1700 & 93 & 5808 & 69 \\
\hline
\end{tabular}


International Journal of Wireless \& Mobile Networks ( IJWMN ), Vol.2, No.3, August 2010

Table 3. The network lifetime and number of failed node when the nodes are heterogeneous

\begin{tabular}{|c|c|c|c|c|c|c|c|c|c|c|c|}
\hline \multicolumn{12}{|c|}{ Heterogeneous } \\
\hline \multicolumn{6}{|c|}{ Short transmission range $(15 \mathrm{~m})$} & \multicolumn{6}{|c|}{ long transmission range $(100 \mathrm{~m})$} \\
\hline $\mathrm{PEC}$ & SIS & $\mathrm{LE}$ & $\overline{\mathrm{CH}}$ & & & $\mathrm{PEC}$ & SIS & $\mathrm{LE}$ & $\overline{\mathrm{CH}}$ & & \\
\hline $\begin{array}{c}\text { Lifeti } \\
\text { me } \\
\text { (Roun } \\
\text { d) }\end{array}$ & $\begin{array}{c}\# \text { OF } \\
\text { FAIL } \\
\text { ED } \\
\text { NOD } \\
\text { ES }\end{array}$ & $\begin{array}{c}\text { Lifeti } \\
\text { me } \\
\text { (Roun } \\
\text { d) }\end{array}$ & $\begin{array}{c}\text { \# OF } \\
\text { FAIL } \\
\text { ED } \\
\text { NOD } \\
\text { ES }\end{array}$ & $\begin{array}{c}\text { Lifeti } \\
\text { me } \\
\text { (Roun } \\
\text { d) }\end{array}$ & $\begin{array}{c}\text { \# OF } \\
\text { FAIL } \\
\text { ED } \\
\text { NOD } \\
\text { ES }\end{array}$ & $\begin{array}{c}\text { Lifeti } \\
\text { me } \\
\text { (Roun } \\
\text { d) }\end{array}$ & $\begin{array}{c}\text { \# OF } \\
\text { FAIL } \\
\text { ED } \\
\text { NOD } \\
\text { ES }\end{array}$ & $\begin{array}{c}\text { Lifeti } \\
\text { me } \\
\text { (Roun } \\
\text { d) }\end{array}$ & $\begin{array}{c}\# \text { OF } \\
\text { FAIL } \\
\text { ED } \\
\text { NOD } \\
\text { ES }\end{array}$ & $\begin{array}{c}\text { Lifeti } \\
\text { me } \\
\text { (Roun } \\
\text { d) }\end{array}$ & $\begin{array}{c}\# \text { OF } \\
\text { FAIL } \\
\text { ED } \\
\text { NOD } \\
\text { ES }\end{array}$ \\
\hline 2482 & 69 & 1418 & 76 & 42 & 4 & 1925 & 100 & 1470 & 95 & 4448 & 2 \\
\hline
\end{tabular}

Table 4. The network lifetime with various numbers of clusters

\begin{tabular}{|l|l|l|l|}
\hline Lifetime & $\begin{array}{l}\text { \# of } \\
\text { cluster } \\
\text { head }\end{array}$ & Leach & VGA \\
\hline \multirow{4}{*}{$\begin{array}{l}\text { Loss of } \\
\text { connectivity }\end{array}$} & $25 \%$ & 365 & 2338 \\
\cline { 2 - 4 } & $16 \%$ & 419 & 2268 \\
\cline { 2 - 4 } & $9 \%$ & 1116 & 1142 \\
\cline { 2 - 4 } & $4 \%$ & 2497 & 723 \\
\hline
\end{tabular}

\section{Conclusion}

In this paper, we analyzed three different types of hierarchical routing protocols: PEGASIS, LEACH and VGA. Sensoria simulator is used to compare the performance of the three routing Protocols.Using PEGASIS can greatly prolong sensor network's life time when the transmission range is limited. VGA saves more energy than other protocols when the transmission range is longer. Using homogeneous nodes are better than heterogeneous nodes with all routing protocols. The network lifetime increases as the number of clusters decreases

\section{ACKNOWLEDGEMENTS}

We thank Dr. Jamal N. Al-karaki and Ghada A. Al-Mashaqba to provide us with the Sensoria simulator, open source version.

\section{REFERENCES}

[1] Deborah Estrin, Ramesh Govindan, John S. Heidemann, and Satish Kumar. Next century challenges: Scalable coordination in sensor networks. In Mobile Computing and Networking, pages 263-270, Seattle, WA USA, 1999.

[2] G. J. Pottie and W. J. Kaiser. Wireless integrated network sensors. Commun. ACM, 43(5):51-58, 2000. 
[3] L. Subramanian and R. H. Katz: An Architecture for Building Self Configurable Systems, Proc. IEEE/ACM Wksp. Mobile Adhoc Net. and Comp., Boston, MA ( Aug. 2000).

[4] J. Pan, L. Cai, T. Hou, Y. Shi, and S. Shen: Topology control for wireless sensor networks, Proceedings of the Nineth ACM MobiCom, (2003).

[5] J.N Al-Karaki, and A.E. Kamal, "Routing techniques in wireless sensor networks: a survey", IEEE Wireless Communications, Vol. 11, No. 6, pp.6-28, December 2004.

[6] W. Heinzelman, A. Chandrakasan and H. Balakrishnan, "Energy-efficient Communication Protocol for Wireless Mi- crosensor Networks," Proceedings of the 33rd Hawaii International Conference on System Sciences (HICSS '00), January 2000.

[7] S. Lindsey, C. Raghavendra, IPEGASIS: Power-Efficient Gathering in Sensor Information Systems", IEEE Aerospace Conference Proceedings, 2002, Vol. 3, 9-16 pp. 1125-1130.

[8] Stephanie Lindsey, Cauligi S. Raghavendra, "PEGASIS Power-Efficient Gathering in Sensor Information System," IEEE Aerospace Conference Proceedings 2002, Volume 3, pp.1125-1130, 2002.

[9] Kemei Du, Jie Wu, and Dan Zhou, "Chain-based Protocols for Data Broadcasting and Gathering in the Sensor Networks", Parallel and Distributed Processing Symposium, Apr. 2003.

[10] J. N. Al-Karaki, A. E. Kamal, "End-to-End Support for Statistical Quality of Service in Heterogeneous Mobile Ad hoc Networks", Journal on Computer Communications, accepted for publication

[11] J. N. Al-Karaki, and G. A. Al-Mashaqbeh, "SENSORIA: A New Simulation Platform for Wireless Sensor Networks", International Conference on Sensor Technologies and Applications, 2007.

[12] R. V. Biradar, V. C. Patil, Dr. S. Sawant, and Dr. R. R. Mudholkar, "Classification and comparisson of routing protocols in wireless sensor networks", UbiCC Journal, Vol. 4.

[13] http://alkautsarpens.wordpress.com/wsn/

[14] M.A. Abuhelaleh T. M. Mismar and A. A. Abuzneid," Armor-LEACH - Energy Efficient, Secure Wireless Networks Communication “,IEEE 2008

\section{Authors}

Amer Al-Rahayfeh is a Ph.D. student of Computer Science and Engineering at the University of Bridgeport. He received a B.S. in Computer Science from Mutah University and an M.S.in Computer Information Systems from The Arab Academy for Banking and Financial in 2002 and 2004. He worked as an Instructor at The Arab Academy for Banking and Financial (2006-2008), Amer current research interests are in multimedia database systems.

Muder Almi'ani is a Ph.D. student of Computer Science and Engineering at the University of Bridgeport. He received a B.S. in Computer Science from Al-Hussein Bin Talal University and an M.S.inComputer Information Systems from The Arab Academy for Banking and Financial in 2004 and 2008 . He worked as an Instructor at The Al- Balqa' Applied University (2004-2008), Muder current research interests are in Database management systems .

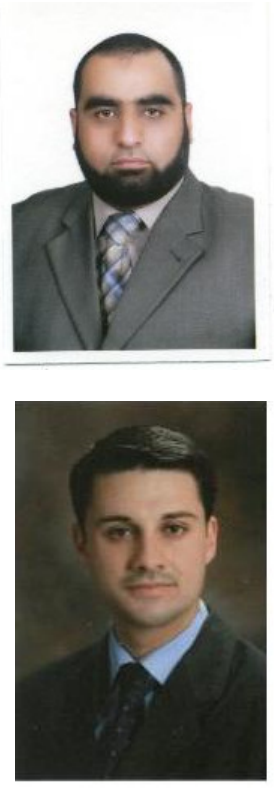


International Journal of Wireless \& Mobile Networks ( IJWMN ), Vol.2, No.3, August 2010

Abdelshakour Abuzneid has received his BS degree in Computer Engineering and Control from Yarmouk University and MS degree in Computer Engineering from the University of Bridgeport in May 2007. Currently he is pursuing his PhD in Computer Science \& Engineering from the University of Bridgeport. His research interest is in Data / computer / wireless / mobile communications. He has published few journal and conference papers.

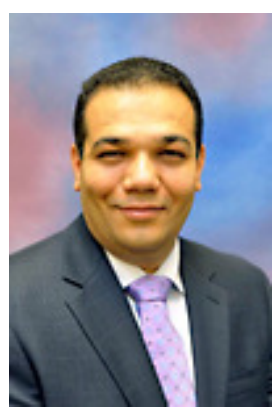

